\section{Lesinurad combination therapy with allopurinol in gout: do CLEAR studies make the treatment of gout clearer?}

\author{
Jasvinder A Singh ${ }^{1,2,3}$
}

Gout is an often forgotten disease, ${ }^{1}$ despite being the most common inflammatory arthritis in adults in the Western world. ${ }^{2-4}$ Lesinurad, a new urate-lowering therapy (ULT), is now approved for the treatment of gout in the USA and the European Union, ${ }^{5}$ and is in phase III programmes as a combination therapy in many other countries. Lesinurad is a selective inhibitor of urate/anion exchanger 1 and organic acid transporter 4, two urate transporters responsible for the reabsorption of urate from the proximal renal tubule. ${ }^{7}$ Probenecid and benzbromarone are the other uricosurics available for use in gout as monotherapy or combination with allopurinol in case of an inadequate response to allopurinol. In the current issue of the journal, Bardin et al present the results of a 12-month, randomised, phase III trial efficacy and safety of oral lesinurad (200 or $400 \mathrm{mg}$ ) in combination with allopurinol. ${ }^{8}$ Combining Lesinurad with Allopurinol in Inadequate Responders (CLEAR) were two replicate studies, one in the USA (CLEAR- 1$)^{9}$ and one in Europe (CLEAR-2), published in the current issue of this journal. ${ }^{8}$ CLEAR-2 compared daily lesinurad (200 or $400 \mathrm{mg}$ orally) with placebo when added to allopurinol in 610 patients with gout with serum uric acid (sUA) above target $(<6 \mathrm{mg} / \mathrm{dL}$ or $<0.36 \mathrm{mmol} / \mathrm{L}$ ) and frequent gout flares ( $\geq 2$ gout flares in the prior year).

\section{WHAT DID THE CLEAR-2 TRIAL RESULTS SHOW?}

The primary trial endpoint of sUA $<6 \mathrm{mg} / \mathrm{dL} \quad$ (ie, $\quad<0.36 \mathrm{mmol} / \mathrm{L}$ ) at 6 months was achieved by a significantly greater proportion of patients, $55 \%$ and $66 \%$ in the lesinurad $200 \mathrm{mg}+$ allopurinol and lesinurad $400 \mathrm{mg}+$ allopurinol groups versus $23 \%$ in the allopurinol alone group

${ }^{1}$ Birmingham VA Medical Center, Birmingham,

Alabama, USA; ${ }^{2}$ Department of Medicine at the School of Medicine, University of Alabama at Birmingham, Birmingham, Alabama, USA; ${ }^{3}$ Division of Epidemiology at the School of Public Health, University of Alabama at Birmingham, Birmingham, Alabama, USA

Correspondence to Dr Jasvinder A Singh, University of Alabama, Faculty Office Tower 805B, 510 20th Street S, Birmingham, AL 35294, USA; Jasvinder.md@gmail.com $\left(\mathrm{p}<0.0001\right.$ vs either lesinurad group) ${ }^{8}$ These rates were similar to the achievement of target sUA $<6 \mathrm{mg} / \mathrm{dL}$ (ie, $<0.36 \mathrm{mmol} / \mathrm{L}$ ) in CLEAR-1.9 To my knowledge, CLEAR $-2^{8}$ and the replicate study, CLEAR- $1,{ }^{9}$ are the two largest randomised control trials (RCTs) of lesinurad versus placebo in patients with symptomatic gout with frequent flares despite treatment with allopurinol.

Harms were similar in the lesinurad $200 \mathrm{mg}$ versus placebo groups, but somewhat higher in the lesinurad $400 \mathrm{mg}$ group. Differences were noted in the safety profile. Serious adverse events and renal adverse events occurred in similar proportions of patients receiving the lesinurad $200 \mathrm{mg}+$ allopurinol and placebo + allopurinol group (4-6\%), but in 2-3 times as many people in the lesinurad $400 \mathrm{mg}$ +allopurinol group, with incidences of $10 \%$ and $15 \%$, respectively. Among renal adverse events, increased serum creatinine was seen in same proportions of the lesinurad $200 \mathrm{mg}+$ allopurinol and allopurinol-only groups, but in twice as many people in the higher lesinurad group. Renal failure and serum creatinine elevations of $\geq 1.5$ times baseline value were more frequent with both lesinurad $200 \mathrm{mg}+$ allopurinol and lesinurad $400 \mathrm{mg}+$ allopurinol groups compared with allopurinol alone. These serum creatinine elevations were mostly reversible, except in seven patients in the lesinurad $400 \mathrm{mg}$ dose and three patients in the allopurinol group; similarly, five cases of serum creatinine elevations of $\geq 2$ times baseline value were unresolved in the lesinurad $400 \mathrm{mg}$ dose versus none in the other groups by the last study visit.

Evidence of the two studies, CLEAR-1 and CLEAR-2, indicates that compared with placebo plus allopurinol, lesinurad $200 \mathrm{mg}$ /day in combination with allopurinol median dose of $300 \mathrm{mg} /$ day was efficacious and was not associated with a significant increase in rate of serious adverse events or renal adverse events. Lesinurad $400 \mathrm{mg} /$ day in combination with allopurinol is effective, but was associated with clinically meaningfully higher rates of serious adverse events and renal adverse events and possibly renal failure.

\section{WHAT CONCERNS REMAIN?}

Allopurinol was used in 'standard-of-care' doses in patients with gout who were randomised in CLEAR-1, with a mean allopurinol dose of approximately $300 \mathrm{mg} /$ day, similar to that reported in observational cohorts. ${ }^{10} 11$ This allopurinol dose achieves target sUA $<6 \mathrm{mg} / \mathrm{dL}$ in only $50 \%$ of the patients, ${ }^{10} 11$ meaning that the standard allopurinol dose is suboptimal in half of the patients with gout. Allopurinol dose had not been titrated to a maximum approved dose of $800 \mathrm{mg}$ daily (or $900 \mathrm{mg}$ approved maximum dose in Europe) to achieve target sUA in CLEAR studies. The CLEAR-1 and CLEAR-2 studies show that in patients with gout who have failed a suboptimal 100-300 mg daily dose of allopurinol, lesinurad is more beneficial than placebo in achieving target sUA. These studies provide us with the first glimpse of benefits and harms of lesinurad compared with placebo. Perhaps a more informative study for clinicians would have been the use of new therapies (such as lesinurad) in patients who had truly been refractory to allopurinol, that is, failed to achieve a target sUA $<6 \mathrm{mg} / \mathrm{dL}$ despite adequate allopurinol doses titrated to $800 \mathrm{mg}$ of allopurinol or more and used for an adequate duration of treatment (6-12 months). Pharmaceutical companies in the field of developing urate-lowering therapies would be better off planning and conducting trials targeting these true allopurinol refractory patient populations. ${ }^{12}$ Rheumatologists have started using the therapeutic doses of allopurinol up to the approved maximum doses $(800 \mathrm{mg} /$ day in the USA and $900 \mathrm{mg} /$ day in Europe) in their current practice. I hope that this practice of the use of titrated therapeutic allopurinol doses will spread to internists, cardiologists, nephrologists, podiatrists and other physician colleagues, with proper education efforts.

Serum creatinine elevations $\geq 2$ times baseline levels resolved in all patients in the lesinurad $200 \mathrm{mg}+$ allopurinol group but were unresolved in five patients in the lesinurad $400 \mathrm{mg}+$ allopurinol group in CLEAR $-2^{8}$ and unresolved in two cases in the lesinurad $400 \mathrm{mg}+$ allopurinol group in CLEAR-1. ${ }^{9}$ Not surprisingly, in CLEAR-1, renal failure occurred in $1 \%$ and $1.5 \%$ of patients receiving lesinurad 200 and $400 \mathrm{mg}$ versus $0.5 \%$ in placebo, respectively. This is clinically relevant. The absolute risk difference for renal failure with lesinurad $400 \mathrm{mg}$ daily versus placebo was $1 \%$ and the relative risk was approximately three times. Was there anything peculiar about these patients 
regarding comorbidity and/or concomitant medications? Can the clinician be better guided as to who these patients might be so that they can avoid the higher dose of lesinurad in these patients or try some other strategy? It would be very informative to see detailed characteristics of these patients in subsequent publication (s) so that clinicians and patients are better informed about who might be at a higher risk of an irreversible creatinine elevation or renal failure with the higher lesinurad dose.

\section{WAS THERE ANY EVIDENCE OF DIMINISHING CLINICAL RESPONSE OVER TIME? WHAT ELSE DOES THIS TRIAL TEACH US?}

The success rates for achieving target sUA $<6 \mathrm{mg} / \mathrm{dL}$ with lesinurad co-therapy at doses 200 and $400 \mathrm{mg} /$ day were $55 \%$ and $65 \%$ at 6 months compared with $22 \%$ with placebo; proportions were $49 \%$ versus $55 \%$ versus $26 \%$ at 12 months, showing slight loss of efficacy in lesinurad arms. The dropout rate was nondifferential between 6 and 12 months between arms and minimal, 4 versus 1 versus 5 people, respectively. Most patients dropped out before the 6-month time point at $21 \%$ and $25 \%$ versus $23 \%$, respectively. Thus, the slight loss in efficacy in lesinurad arms at 12 months is unlikely to be explained by loss to follow-up. Whether a 6-10\% difference in proportions with target sUA between 6 and 12 months is a chance finding or a reduction of lesinurad efficacy or adherence remains to be seen.

Even though the primary sUA outcome was significantly better in the lesinurad groups compared with placebo, the tophi resolution rate was similar to that observed in the placebo group $-31 \%$ and $28 \%$ versus $33 \%$ had complete resolution of $\geq 1$ target tophi. Was the study too short or patients too few to show differences in tophi resolution? Is the tophus measure not sensitive to change in this patient population? Certainly, tophi were secondary outcome, and therefore, the current study was not powered to detect significant between-group differences. Studies that recruit a larger number or proportion of gout patients with tophi are needed to demonstrate whether tophi resolution is faster in patients with lesinurad compared with placebo. I suspect that such study will be of longer duration since tophi size reduction and resolution cannot be expected in a short duration efficacy trial.

An interesting study finding was that up to a third of the patients treated with allopurinol alone had resolution of $\geq 1$ target tophi, demonstrating that allopurinol is effective, even when used in suboptimal doses. This finding is similar to $50 \%$ reduction in tophus area with allopurinol dose of $200-300 \mathrm{mg}$ daily in another RCT. ${ }^{13}$ If allopurinol $300 \mathrm{mg}$ daily dose, which is effective in achieving target sUA in $50 \%$ of the patients, leads to $29-50 \%$ reduction in target tophi, how much reduction will one expect with a $100 \%$ effective dose? Is it possible that we already have a cure for gout and just have not realised it yet? I suspect that if we use allopurinol at an optimal final dose (up to $800 \mathrm{mg} /$ day in the USA; $900 \mathrm{mg} /$ day in Europe) uptitrated from a lower, starting dose to achieve target sUA, it will be an extremely effective tool both for lowering sUA and resolving tophi. Of course, availability of other ULTs such as febuxostat, pegloticase, traditional uricosurics and now lesinurad, where titrated allopurinol does not succeed or is contraindicated, can help improve our success rate of curing gout.

Importantly, and quite interestingly, a very small proportion of patients $(<15 \%)$ had gout flares requiring treatment since all received anti-inflammatory prophylaxis, which started before the initiation of lesinurad or placebo, and continued for 5 months. The proportion with gout flares did not differ across the lesinurad versus placebo groups. ULT initiation without an anti-inflammatory prophylaxis is associated with frequent gout flares. This study demonstrates that antiinflammatory prophylaxis for gout flares is effective in preventing acute flares if started early and taken for a few months when an ULT is started.

The 25\% discontinuation rate in this published study, ${ }^{8}$ although similar to the $33 \%$ discontinuation rate in a pivotal RCT of febuxostat, ${ }^{13}$ is still higher than the acceptable $20 \%$ dropout rate in trials. A high dropout rate, defined as $>20 \%$, puts the RCT results at a higher risk of bias, ${ }^{14}$ a study limitation that must be considered while interpreting the trial results and making conclusions.

\section{HOW WILL LESINURAD'S AVAILABILITY CHANGE THE MANAGEMENT OF GOUT?}

I think this is the golden era for the treatment of gout. New therapies are being approved and becoming available for the treatment of hyperuricemia and acute gout flares. Investigations of new mechanisms for urate lowering reflect a better understanding of urate metabolism and novel pathways.
Lesinurad's availability offers a new choice of a uricosuric ULT co-therapy option to be used concomitantly with allopurinol for patients with refractory gout despite use of allopurinol. Interestingly, gout flares and tophi resolution were not significantly different from placebo in this 12-month study, but this may be related to small sample size, adequate anti-inflammatory prophylaxis or a small effect size with lesinurad. Based on the evidence, one can consider lesinurad $200 \mathrm{mg} /$ day as a second-line therapeutic option to be co-administered with allopurinol after an adequately titrated dose of allopurinol (approved up to $800 \mathrm{mg} /$ day in the USA and $900 \mathrm{mg} /$ day in Europe) has been tried and fails to control sUA, frequent flares or tophi.

\section{WHAT SHOULD A CLINICIAN} MONITOR WHILE TREATING PATIENTS WITH LESINURAD?

The data presented in the current study and CLEAR-1 provide confidence regarding the risk/benefit ratio of lesinurad $200 \mathrm{mg}$ daily dose in combination with allopurinol. However, in patients without a normal renal function, one needs to cautiously evaluate and discuss the risk/ benefit ratio of lesinurad $400 \mathrm{mg}$ in combination with allopurinol, given a higher risk of serious adverse events and renal adverse events, including renal failure, compared with placebo in both CLEAR-1 and CLEAR-2. It seems that regular monitoring of renal function is prudent when starting lesinurad.

\section{WHAT IS THE TAKE HOME MESSAGE?}

CLEAR- 1 and CLEAR-2 studies bring new knowledge for clinicians and a new treatment for patients. First, lesinurad $200 \mathrm{mg}$ in combination with allopurinol is an effective and safe option for patients with symptomatic gout despite an average allopurinol dose of $300 \mathrm{mg} /$ day. Second, this 12-month study of lesinurad showed an important improvement in sUA target achievement compared with placebo, but no significant difference in gout flares or gouty tophi resolution, both of which were infrequent in this patient population; this indicated that studies with a larger sample size, longer follow-up or that recruit patients with higher baseline tophi are needed. Third, lesinurad's $400 \mathrm{mg}$ daily dose in combination with allopurinol has a different safety profile than the $200 \mathrm{mg}$ daily dose and placebo, characterised by higher rates of serious adverse events, sustained serum creatinine elevations and renal failure. Fourth, allopurinol alone even in average doses of $300 \mathrm{mg}$ daily 
leads to complete resolution of $\geq 1$ target tophi in $29 \%$ of patients. This last message is sentinel, indicating that if we use appropriate doses of allopurinol, we are likely to not only improve gout management and resolve tophi, but also find a better use of newer therapies such as lesinurad and febuxostat and several more to come.

We need more data and longer follow-up studies of lesinurad (some of which are underway) and, in addition, studies that use optimal titrated doses of allopurinol at baseline that achieve target sUA. More comparative effectiveness studies comparing lesinurad to existing uricosurics will also help us better understand when best to use lesinurad versus probenecid or benzbromarone (where available). I hope that future lesinurad studies will use the therapeutic doses of allopurinol $(800 \mathrm{mg} / \mathrm{day}$ in the USA; $900 \mathrm{mg} /$ day in Europe), which are $>300 \mathrm{mg}$ daily in $>50 \%$ of patients with gout, and not the suboptimal 'current standard' allopurinol $300 \mathrm{mg} /$ day doses.

Competing interests JAS has received research grants from Takeda and Savient and consultant fees from Savient, Takeda, Regeneron, Merz, Iroko, Bioiberica, Crealta and Allergan pharmaceuticals, WebMD, UBM LLC and the American College of Rheumatology. JAS serves as the principal investigator for an investigator-initiated study funded by Horizon pharmaceuticals through a grant to DINORA, a 501 (c) (3) entity. JAS is a member of the executive of OMERACT, an organisation that develops outcome measures in rheumatology and receives arms-length funding from 36 companies; a member of the ACR's Annual Meeting Planning Committee; Chair of the ACR Meet-the-Professor, Workshop and Study Group Subcommittee; and a member of the Veterans Affairs
Rheumatology Field Advisory Committee. JAS is supported by the resources and the use of facilities at the VA Medical Center at Birmingham, Alabama, USA.

Provenance and peer review Commissioned; externally peer reviewed.

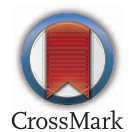

To cite Singh JA. Ann Rheum Dis 2017;76:779-781.

Received 3 October 2016

Revised 12 November 2016

Accepted 8 December 2016

Published Online First 30 December 2016

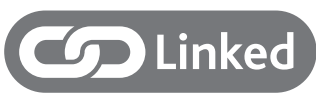

http://dx.doi.org/10.1136/annrheumdis-2016209213

Ann Rheum Dis 2017;76:779-781.

doi:10.1136/annrheumdis-2016-210519

\section{REFERENCES}

1 Lopez AD, Williams TN, Levin A, et al. Remembering the forgotten non-communicable diseases. BMC Med 2014;12:200.

2 Zhu Y, Pandya BJ, Choi HK. Prevalence of gout and hyperuricemia in the US general population: The National Health and Nutrition Examination Survey 2007-2008. Arthritis Rheumatol 2011:63:3136-41.

3 Kuo CF, Grainge MJ, Zhang W, et al. Global epidemiology of gout: prevalence, incidence and risk factors. Nat Rev Rheumatol 2015;11:649-62.

4 Dehlin M, Drivelegka $P$, Sigurdardottir $\mathrm{V}$, et al. Incidence and prevalence of gout in Western Sweden. Arthritis Res Ther 2016;18:164.

5 FDA News Release. FDA approves Zurampic to treat high blood uric acid levels associated with gout.
2015 (cited 10/01/2016). http://www.fda.gov/ NewsEvents/Newsroom/PressAnnouncements/ ucm 478791.htm

6 European Medicines Agency. Zurampic. Lesinurad. 2016 (cited 10/01/2016). http://www.ema.europa. eu/ema/index.jsp?curl=pages/medicines/human/ medicines/003932/human_med_001963. jsp\&mid=WC0b01ac058001d124

7 So A, Thorens B. Uric acid transport and disease. J Clin Invest 2010;120:1791-9.

8 Bardin T, Keenan RT, Khanna PP, et al. Lesinurad in combination with allopurinol: a randomised, double-blind, placebo-controlled study in gout patients with inadequate response to standard of care (the multinational CLEAR 2 study). Ann Rheum Dis 2017;76:811-20.

9 Saag KG, Fitz-Patrick D, Kopicko J, et al. Lesinurad combined with allopurinol: randomized, double-blind, placebo-controlled study in gout subjects with inadequate response to standard of care allopurinol (A US-based Study). Arthritis Rheumatol 2016. doi: 10.1002/art.39840. [Epub ahead of print 26 Aug 2016].

10 Sarawate CA, Patel PA, Schumacher HR, et al. Serum urate levels and gout flares: analysis from managed care data. I Clin Rheumatol 2006;12: 61-5.

11 Singh JA, Hodges JS, Asch SM. Opportunities for improving medication use and monitoring in gout. Ann Rheum Dis 2009;68:1265-70.

12 Rees F, Jenkins W, Doherty M. Patients with gout adhere to curative treatment if informed appropriately: proof-of-concept observational study. Ann Rheum Dis 2013;72:826-30.

13 Becker MA, Schumacher HR Jr, Wortmann RL, et al. Febuxostat compared with allopurinol in patients with hyperuricemia and gout. N Engl I Med 2005;353:2450-61.

14 Higgins JP, Altman DG. Chapter 8: assessing risk of bias in included studies. In: Higgins JPT, Green S, ed. Cochrane handbook for systematic reviews of interventions version 510 (updated March 2011). The Cochrane Collaboration, 2011. http://www. cochrane-handbook.org 